\title{
Improvement of Mechanical Properties of Polymer-clay Nancomposite Hydrogels by Graphene Oxide
}

\author{
M. ZHANG, F.Y. FU \& H.J. LI* \\ School of Chemical Engineering and Environment, Beijing Institute of Technology, Beijing 100081, China
}

\begin{abstract}
Poly (N-isopropylacrylamide) (PNIPAm) hydrogels have been proposed for application as promising biomaterials and stimuli-responsive materials in biomedical fields, but their poor mechanical properties limited their development. Here we report that the mechanical properties of PNIPAm-clay nanocomposite hydrogels were enhanced significantly through in situ polymerization by addition of very low content of graphene oxide (GO). GO sheets were used as both the crosslinking agent and reinforcing agent. Compared to nanocomposite hydrogels without GO, a more than $200 \%$ increase in tensile strength and a nearly $400 \%$ improvement of Young's modulus were achieved with the addition of $0.1 \mathrm{wt}$ \% of GO, which suggests an excellent load transfer between the GO and hydrogel matrix.
\end{abstract}

KEYWORD: Graphene oxide; hydrogel; N-isopropylacrylamide

\section{INTRODUCTION}

Poly (N-isopropylacrylamide) (PNIPAm) hydrogel is one of non-ionic temperature sensitive hydrogels with lower critical solution temperature (LCST) of $33{ }^{\circ} \mathrm{C}$ (Cong et al 2014). When the ambient temperature changing around the LCST, the performance of PNIPAm hydrogel responses rapidly (Yuan et al 2014). This property makes it an excellent candidate to bio-medical applications such as therapeutic agent delivery systems, bio-separation devices, tissue engineering scaffolds and so on (Vagias et al 2014). Unfortunately, the conventional PNIPAm hydrogel is brittle with poor mechanical property which significantly limits its application. Therefore, much attention has been focused on the modification of pure PNIPAm hydrogel ( $\mathrm{Li}$ et al 2014).

Recently, Haraguchi (Haraguchi et al 2003) have proposed the method of using inorganic clay (Clay) instead of organic crosslinking agent to prepare hydrogels with improved mechanical performance. In their opinion, Clay and monomers form nanocomposite hydrogel by in situ polymerization. Exfoliated clay platelets play the role of crosslinking agent by hydrogen bond, ionic bond and ligand bond (Haraguchi et al 2006, Farnworth, R. et al 2003\& Haraguehi et al. 2002). These nanocomposite hydrogels have high tensile strength and good toughness (Chen et al 2000).
On the other hand, GO, because of outstanding mechanical and thermal properties, has attracted considerable attention (Liu et al 2013). GO with large specific surface area and carboxyl, hydroxyl and epoxy is able to disperse in many monomers and produce lots of functional hydrogels (Liu et al 2012). GO can be physical cross-linker and enhancing agent (Li et al 2013). Reported by L Zhang,( Zhang et al 2011) compared to pure PVA hydrogels, a $132 \%$ increase in tensile strength and a $36 \%$ improvement of compressive strength were achieved with the addition of $0.8 \mathrm{wt} \%$ of $\mathrm{GO}$, which suggests an excellent reinforcement between the GO and the PVA matrix. Namely, some addition of GO is helpful for the preparation of high strength hydrogels (Wang et al 2010).

Here we first proposed a simply strategy by using graphene oxide both as crosslinking agent and enhancing agent to prepare graphene oxide/clayPNIPAm hydrogel (NC-GO hydrogel) by in situ polymerization. The obtained nana- composite hydrogel exhibited a significant improvement of mechanical strength by the addition of $0.1 \mathrm{wt} . \%$ of GO.

\section{RESULTS AND DISCUSSION}

\subsection{The AFM images of exfoliated graphene oxide}

The AMF images of GO is shown in Fig. 1. As could be seen, the GO nanosheets were of irregular shape 
with uniform thickness $(\sim 1.1 \mathrm{~nm})$ according to cross-section analysis and lateral dimensions ranging from several hundred nanometers to several micrometers. While individual graphene nanosheet is atomically flat with a well-known thickness of $\sim 0.34 \mathrm{~nm}, \mathrm{GO}$ is much thicker due to the attachment of oxygen-containing functional groups such as epoxy, hydroxyl and carboxyl groups, which disrupt the sp2-bonding nature of carbon atoms and introduce structure defects to form folds on the GO sheets. The results are consistent with those reported in the literature (Haraguchi et al 2006), which proving the graphene oxide is capable of forming uniform dispersion of single thickness sheet.

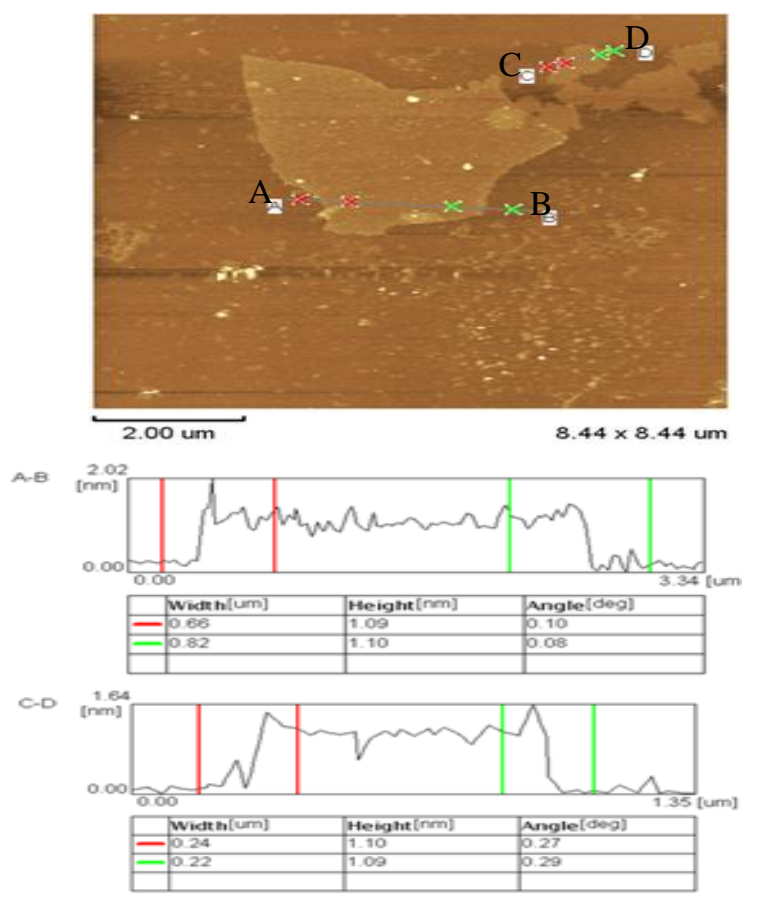

Figure 1. The AFM images of graphene oxide

\subsection{The mechanical properties of NC-GO hydrogels}

\subsubsection{The images of NC and NC-GO hydrogels}

It can be observed from Fig. 2a and Fig. 2b, compared to $\mathrm{NC}$ hydrogel, NC-GO hydrogel has better strength and toughness. GO has high specific surface energy, good hydrophilicity, good mechanical properties, and can be well dispersed in water. Composites consisting of GO and polymer can realize the complementary of the two components which result in mechanical enhancement. Since the excellent mechanical properties of graphene oxide, it is considered desirable reinforcing fillers in polymer composites (Wang et al 2010). In the process of forming the hydrogel, the epoxy, hydroxyl and carboxyl groups on the edge of GO with the polymer chains form dense three-dimensional network structure which is crucial for the improvement of the mechanical properties.
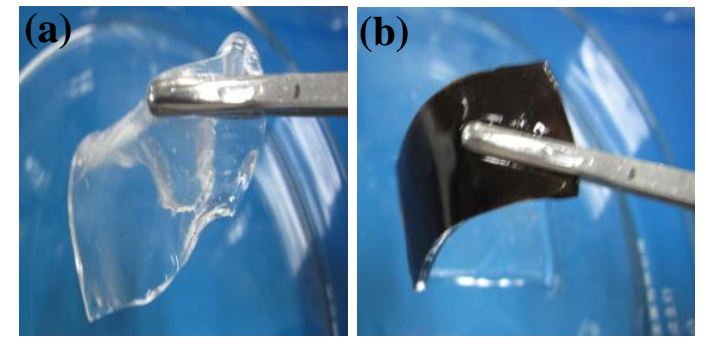

Figure 2. The photographs of Clay- PNIPAm (NC) hydrogel (a) and NC-GO hydrogel (b)

\subsubsection{Mechanical behavior}

Fig. 3 shows the stress-strain curves of NC hydrogel with different content of GO. One can see that the addition of GO significantly improved the tensile properties of NC hydrogels. The tensile strength and Young's modulus increased sharply by more than $200 \%$ from $50 \mathrm{KPa}$ to $111 \mathrm{KPa}$ (Figure 3a) and by nearly $400 \%$ from $5.6 \mathrm{KPa}$ to $20.4 \mathrm{KPa}$, respectively. The addition of GO made the mechanical property of the hydrogel enhanced dramatically due to the increasing of crosslinking points. On the other hand, the hydrogen bond between epoxy, hydroxyl and carboxyl groups on GO made the hydrogel network more dense which led to the improvement of tensile strength. It was strongly obvious that even a small amount of GO could significantly improve the mechanical properties (Fig. 3b). The content of GO increased from $0.01 \mathrm{wt} \%$ to $0.1 \mathrm{wt}$. $\%$, the tensile strength increased with the increasing of GO. Surprisingly, when the content of GO increased from 0.1 wt. $\%$ to 0.15 wt. $\%$, the tensile strength decreased on the contrary. This may be caused by excessive crosslinking points of excessive GO, thus affecting the mechanical properties of the entire system.
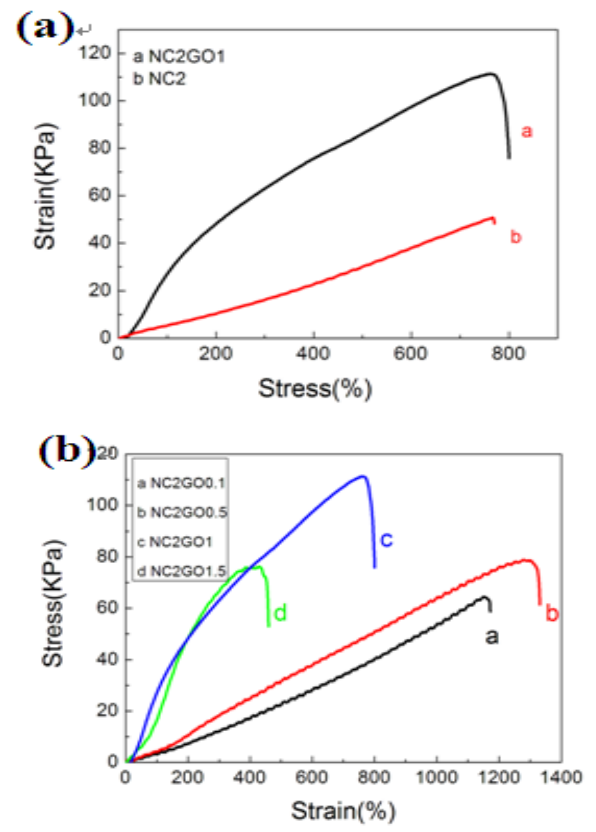

Figure 3. The stress-strain curves of NC2 hydrogel and NC2GO1 hydrogel (a) and NC2-GO hydrogels with different contents of GO (b) 


\section{CONCLUSIONS}

Graphene oxide/clay-PNIPAm nanocomposite hydrogels were prepared by in situ free-radical polymerization. The mechanical strength dramatically enhanced by the addition of small amount of GO. The stress and strain of the hydrogels gradually increased then decreased with increasing GO content. The tensile strength and Young's modulus increased sharply by more than $200 \%$ from $50 \mathrm{KPa}$ to $111 \mathrm{KPa}$ and by nearly $400 \%$ from 5.6 $\mathrm{KPa}$ to $20.4 \mathrm{KPa}$ with the content of GO of 0.1 wt.\%, respectively. This finding has provided a simple method to enhance mechanical properties of hydrogels for biomedical applications such as artificial cartilages, artificial muscles, tissue engineering.

\section{REFERENCES}

[1] Cong, H.L. 2014. Poly (N-isopropylacrylamide) -blockpoly (acrylic acid) hydrogels: synthesis and rapid thermoresponsive properties. Colloid Polym Sci 292(2): 2633-2645.

[2] 2 Yuan, H.H. 2014. Regulating drug release from $\mathrm{pH}$ - and temperature-responsive electrospun CTS-gPNIPAAm/poly (ethylene oxide) hydrogel nanofibers. Biomedical Materials 9: 335-346.

[3] Vagias, A. 2014. Dynamics in stimuli responsive Poly (Nisopropylacrylamide) hydrogel layers as revealed by fluorescence correlation spectroscopy. Macromolecules 47(5): 5303-5312.

[4] Li, X. 2014. A PNIPAAm-based thermosensitive hydrogel containing SWCNTs for stem cell transplantation in myocardial repair. Biomaterials 35: 5679-5688.
[5] Haraguchi, K. 2003. Compositional effects of mechanical properties of nanocomposite hydrogels composed of poly(N,N'-dimethylacrylamide) and clay. Macromolecules 36: 5732-5741.

[6] Haraguchi, K. 2006. Mechanical properties and structure of polymer-clay nanocomposite gels with high clay content. Macromolecules 39: 1898-1905.

[7] Farnworth, R. 2003. Compositional effects on mechanical properties of nanocomposite hydrogels composed of Poly (N,N-dimethylacrylamide) and clay. Macromolecules 36: 5732-5741.

[8] Haraguehi, K. Takehisa, T. 2002. Nanocomposite hydrogels: a unique organic-inorganicnetwork structure with extraordinary mechnaical and swelling/deswelling Properties. Advanced Material 14: 1120-1124.

[9] Chen, L. 2000. Effects of polyelectrolyte complexation on the UCST of zwitterionic polymer. Polymer 41: 141147.

[10] Liu, Z. 2013. Lyotropic liquid crystal of polyacrylonitrilegrafted graphene oxide and its assembled continuous strong nacre-mimetic fibers. Macromolecules 46:6931-6941.

[11] Liu, R.Q. 2012. Tough and highly stretchable graphene oxide/polyacrylamide nanocomposite hydrogels. Journal of Materials Chemistry 22: 14160-14167.

[12] Li, H. 2013. Strong compositefilms with layered structures prepared by casting silkfibroin-graphene oxide hydrogels. Nanoscale 5: 3780-3786.

[13] Zhang, L. 2011. High strength graphene oxide/polyvinyl alcohol composite hydrogels, Journal of Materials Chemistry 21: 10399-10406.

[14] Wang, X.L. 2010. Electrically conductive and mechanically strong biomimetic chitosan/reduced graphene oxide composite films. Journal of Materials Chemistry 20: 9032-9036. 